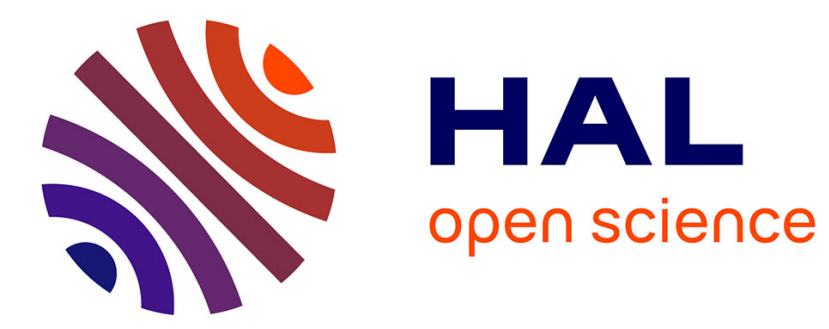

\title{
Compactage d'un massif d'agrégats : effet de la teneur en eau et de la pression appliquée
}

\author{
Jérôme Guérif
}

\section{To cite this version:}

Jérôme Guérif. Compactage d'un massif d'agrégats: effet de la teneur en eau et de la pression appliquée. Agronomie, 1982, 2 (3), pp.287-294. hal-00884383

\section{HAL Id: hal-00884383 \\ https://hal.science/hal-00884383}

Submitted on 1 Jan 1982

HAL is a multi-disciplinary open access archive for the deposit and dissemination of scientific research documents, whether they are published or not. The documents may come from teaching and research institutions in France or abroad, or from public or private research centers.
L'archive ouverte pluridisciplinaire HAL, est destinée au dépôt et à la diffusion de documents scientifiques de niveau recherche, publiés ou non, émanant des établissements d'enseignement et de recherche français ou étrangers, des laboratoires publics ou privés. 


\title{
Compactage d'un massif d'agrégats : effet de la teneur en eau et de la pression appliquée
}

\author{
Jérôme GUÉRIF
}

I.N.R.A., Station de Science du Sol, Centre de Recherches agronomiques d'Avignon, Domaine Saint-Paul, F 84140 Montfavet.

\section{RÉSUMÉ}

Cohésion,

Angle de frottement interne,

Compression

Milieu non saturé,

Sol argileux,

Oedomètre.
Le comportement au compactage de massifs d'agrégats $(1-2 \mathrm{~mm})$ est étudié en fonction de 3 variables : la teneur en eau, l'intensité de la pression et le temps d'application de la charge tout en contrôlant l'état initial. L'augmentation de compacité des massifs d'agrégats sous compression est due, en fonction de la teneur en eau, à 3 mécanismes :

- réarrangement des agrégats à l'état solide : le frottement entre les agrégats détermine l'amplitude de la déformation - ils ont un comportement de sables.

- réarrangement des agrégats après rupture d'un certain nombre d'entre eux : leur cohésion est de l'ordre de grandeur des contraintes qui se développent dans le massif lors de la compression.

- réarrangement des agrégats après leur déformation. L'amplitude de la déformation est fonction du temps d'application de la charge.

On montre comment le comportement mécanique d'un massif d'agrégats dépend, en fonction de la teneur en eau, des caractéristiques mécaniques propres à l'assemblage des éléments structuraux et des caractéristiques mécaniques propres à l'assemblage des constituants élémentaires du matériau.

\section{SUMMARY \\ Cohesion, \\ Internal friction angle, \\ Compression, \\ Unsaturated-media, \\ Clay soil, \\ Oedometer.}

\section{Compression of aggregate beds : effect of moisture content and load}

The compressive behaviour of beds of natural aggregates, under uniaxial stress, is investigated according to three variables : moisture content, load intensity and loading-time, after checking the initial void ratio. The increase in compactness of aggregate beds under compression is determined by three mechanisms, depending on moisture content :

i) rearrangement of the aggregates in the solid state; the amplitude of deformation is determined by the friction between aggregates - they behave as sand particles.

ii) some of the aggregates are subject to brittle fracture, material from these flows into the interstices between the remaining intact aggregates.

iii) rearrangement of aggregates occurs by plastic flow. The amplitude of the deformation is time-dependent. Compressive behaviour of aggregate beds is shown to be dependent, according to moisture content, on the mechanical characteristics arising from the packing of the individual mineral particles and on the mechanical characteristics arising from the packing of the aggregates.

\section{INTRODUCTION}

Le comportement au compactage des sols cultivés est, principalement au niveau des couches de surface, un élément essentiel dans le choix des techniques culturales et dans l'évaluation de leurs effets. Certaines techniques de travail du sol ont pour objectif direct de tasser le sol: roulage des semis, pour améliorer le contact terre-graine, correction des terres creuses. Ces techniques, mais aussi de nombreuses autres, à objectifs divers, peuvent conduire à un compactage excessif et involontaire, avec les conséquences défavorables souvent décrites (CHANCELLOR, 1977; ERIKSSON, 1974) sur le comportement de la végétation et sur le rendement de la culture en raison d'une limitation de l'extension du système racinaire et d'une baisse de conductivité hydraulique entrainant une mauvaise aération du profil.
Il est, dans ces conditions, tout à fait indispensable d'avoir une connaissance suffisante du comportement au compactage des terres agricoles pour définir et contrôler les techniques de tassement et limiter les déformations dangereuses consécutives à la mise en œuvre d'itinéraires techniques mal adaptés.

Parmi les nombreux travaux concernant le compactage en Génie Civil, très peu sont, directement du moins, utilisables pour analyser et prévoir le tassement dans les conditions agricoles. Cette constatation est à relier au fait que les hypothèses de continuité, d'isotropie et d'homogénéité, classiquement admises en mécanique des sols, ne sont pas vérifiées dans les couches de surface des sols agricoles.

Ainsi, la position d'interface sol-atmosphère propre aux couches de surface, liée à l'utilisation agricole qui en est faite, leur confère une structure : 
- discontinue du fait du travail du sol, des alternances climatiques et de l'action des systèmes racinaires (individualisation d'éléments structuraux : mottes et agrégats).

- anisotrope du fait des façons culturales (par individualisation d'horizons), des roulages (gradients de compacité) et des transferts d'eau (développement de profils hydriques).

A ces deux caractéristiques du profil vient s'ajouter une hétérogénéité de constitution liée à la présence de matières organiques.

De plus, les conditions de compactage des sols cultivés se différencient de celles des ouvrages du Génie civil par le niveau, généralement plus faible, de l'intensité des pressions exercées et par leur temps court d'application.

La plupart des travaux menés sur le sujet, pour répondre au problème agronomique évoqué ci-dessus, ne sont généralement que des constatations de l'interaction entre les techniques et le tassement observé. Ils tentent d'orienter empiriquement les décisions pratiques comme la pression des pneumatiques, le poids des engins agricoles, le choix des roues : (cages, jumelées, etc...). Mais le plus souvent d'un auteur à l'autre, voire même d'une année sur l'autre pour un même auteur, les indications s'avèrent contradictoires.

Néanmoins, en marge de cette attitude empirique, certains auteurs ont tenté une approche plus analytique :

Ainsi, Faure (1978), avec pour objectif général d'expliciter l'effet de la constitution du matériau et de sa teneur en eau, a mis en évidence l'existence de seuils hydriques de changement de comportement au compactage. Ceux-ci dépendent de l'énergie de compactage et sont statistiquement reliés à la teneur en argile. Il en est de même pour les constituants organiques (GUERIF, 1979 ; GUERIF \& FAURE, 1979).

Mais si la méthode de compactage utilisée (test Proctor) a été particulièrement révélatrice de l'effet de la teneur en eau, en revanche elle est inopérante dans l'analyse des variations de compacité du matériau.

Le test Proctor consiste à remplir de matériau, en 3 couches successives, un moule de volume connu, par compactage avec une dame de masse donnée tombant d'une hauteur donnée, un nombre de fois donné.

Compte tenu de la teneur en eau des échantillons compactés et des masses de matériau que l'on a pu introduire dans le moule, on obtient une série de couples $\left(\rho_{d}, W\right)$. Le tracé de la courbe complète est obtenu en répétant l'opération sur des échantillons de teneur en eau croissante.

Le protocole et l'appareillage de ce test ne permettent :

- ni d'analyser les variations du volume de l'espace poral d'un échantillon, son état initial n'étant pas défini,

- ni de traduire à des fins prédictives ou analytiques l'énergie de compactage en terme de pression appliquée.

Il est donc impossible avec cette méthode :

- d'établir des relations entre pression appliquée et potentiel de l'eau dans le matériau,

- de transposer des résultats acquis au laboratoire dans des analyses de comportements in situ.

Par ailleurs, on note des tentatives de rendre compte, sous forme d'ajustements statistiques, des variations du volume de l'espace poral d'un massif d'agrégats en fonction de la pression appliquée, (SCOTT BLAIR, 1937 ; SCOTT BLAIR \& CASHEN, 1938 ; KUIPERS, 1959).

Le modèle, plus récent, de BRAUNACK \& DEXTER (1978) rend bien compte de l'allure générale du phénomène, mais sa construction empirique, dépourvue d'éléments analyti- ques simples caractérisant le matériau, interdit toute généralisation.

Le travail qui fait l'objet de cet article amorce l'étude des variations du volume poral d'une couche de sol soumise à une pression en fonction des différents comportements du matériau induits par sa teneur en eau et son état initial.

Par rapport au problème présenté plus haut, dans son ensemble, on ne prendra en compte ni les facteurs d'anisotropie du profil, ni l'hétérogénéité de constitution liée à la matière organique.

On a pu montrer, par ailleurs (GUERIF, 1978 ; GUERIF \& FAURE, 1979) comment intervient cette dernière pour diminuer l'amplitude du tassement. Une meilleure connaissance du comportement propre à la fraction minérale est un préalable à tout travail dans ce domaine.

En utilisant une technique de compactage permettant de maîtriser l'intensité et le temps d'application de la pression ainsi que l'état initial de l'échantillon et en prenant en compte la discontinuité du matériau, on montrera plus précisément :

- d'une part, quelle est l'influence de l'intensité de la pression appliquée sur les seuils hydriques de changement de comportement,

- d'autre part, quelles sont les variables qui permettraient d'expliciter les comportements ainsi définis.

\section{MATÉRIEL ET METHODE}

\section{A. Technique de compactage}

Les impératifs de choix de la technique de compactage évoqués dans l'introduction nous ont amené à utiliser l'œdomètre comme appareillage expérimental.

La cellule de compression est constituée d'un cylindre et d'un piston de $7 \mathrm{~cm}$ de diamètre, la hauteur de l'échantillon avant compactage étant de $2,4 \mathrm{~cm}$.

Cette stricte définition du volume initial de l'échantillon permet d'en maîtriser l'état initial.

On applique à l'échantillon un effort vertical monodimensionnel ; l'application statique de l'effort, par opposition à un compactage dynamique comme le Proctor, donne la possibilité de contrôler au mieux l'intensité et le temps d'application de la charge : 8 intensités de pression, compatibles avec les gammes des pressions au sol engendrées généralement lors du passage des engins agricoles, ont été retenues ( $\sigma=0,5-1-2-3-4-6-8-10$ bars) et 2 temps d'application de la pression $\left(t_{1}=4 h ; t_{2}=1 \mathrm{mn}\right)$, le temps $t_{1}$ permettant d'atteindre un état d'équilibre, le temps $t_{2}$ étant choisi, comme très petit par rapport à $t_{1}$, mais suffisamment long pour assurer une bonne répétabilité des résultats. Ce souci élimine a priori un choix de $t_{2}$ proche des temps d'application des pressions observées lors de roulage (temps de contact $1 / 25 \mathrm{~s}$ pour une vitesse d'avancement de $0,8 \mathrm{~m} / \mathrm{s}$ ).

La précision sur le temps $t_{2}$ est obtenue par l'utilisation d'un bâti ædométrique pneumatique (DEvaUD, 1977).

Les variations du volume de l'échantillon ne sont dues qu'à la variation de sa hauteur. Celle-ci est suivie par un capteur de déplacement qui permet d'enregistrer, sur un traceur de courbe, la course du piston (fig. 4).

\section{B. Expression de la compacité}

L'amplitude du tassement d'un massif d'agrégats résulte des effets opposés de l'humidité sur le gonflement du matériau et sur son aptitude à se compacter. 
Pour analyser les variations de compacité d'un massif d'agrégats, en s'affranchissant de leur composante de gonflement, nous avons choisi de dissocier dans l'espace poral du massif :

- l'espace poral inter-agrégats,

- l'espace poral intra-agrégats.

L'espace poral intra-agrégats sera assimilé par la suite à l'espace poral textural du matériau (FIES, 1978 ; STENGEL, 1979). En toute rigueur, ceci conduit à une légère surestimation de l'espace poral textural. Les agrégats choisis, compte tenu de leur texture, sont en effet d'un volume tel qu'ils contiennent une part de microfissuration (FIES \& STENGEL, 1981).

La relation indice des vides des agrégats-teneur en eau (courbe de retrait-gonflement) permet la connaissance, pour toute teneur en eau, de l'état de gonflement du matériau.

L'indice des vides e étant le rapport entre le volume des vides et le volume de solide $: \mathrm{e}=\frac{\rho_{\mathrm{s}}}{\rho_{\mathrm{d}}}-1$

$\rho_{\mathrm{s}}$ étant la masse volumique de solide,

$\rho_{d}$ étant la masse volumique sèche de l'échantillon mesuré au niveau considéré (agrégat ou massif d'agrégat).

L'indice des vides inter-agrégats traduira donc la part de l'espace poral du massif, indépendant de l'état de gonflement du massif et susceptible d'être modifié lors du compactage.

Cet indice des vides inter-agrégats est une bonne approximation de l'indice des vides structural $e_{s}$ (compte tenu des réserves émises sur l'indice de vide textural).

On aura :

$$
\mathrm{e}_{\mathrm{s}}=\mathrm{e}_{\mathrm{T}}-\mathrm{e}_{\mathrm{a}}=\frac{\rho_{\mathrm{s}} \mathrm{V}}{\mathrm{m}}-1-\mathrm{e}_{\mathrm{u}}
$$

où : $\mathbf{e}_{\mathrm{T}}$ est l'indice des vides du massif d'agrégats,

$\mathrm{e}_{\mathrm{a}}$ est l'indice des vides des agrégats,

$\rho_{\mathrm{s}}$ est la masse volumique de solide,

$\mathrm{V}$ le volume du massif d'agrégat,

$\mathrm{m}$ la masse de l'échantillon après séchage à $105^{\circ} \mathrm{C}$.

En choisissant $\mathrm{e}_{\mathrm{s}}$ comme variable pour caractériser l'état de compacité d'un massif discontinu, on a été, tout naturellement, amené à définir l'état initial des massifs d'agrégats par un indice des vides structural initial $\mathrm{e}_{\mathrm{s}}^{0}$ constant quelle que soit sa teneur en eau et correspondant à l'assemblage le plus lâche possible des agrégats. La valeur numérique choisie pour $\mathrm{e}_{\mathrm{s}}^{0}$ étant l'indice des vides inter-agrégats moyen (10 répétitions) de massifs d'agrégats secs mis en place par écoulement, aussi ménagé que possible, dans un volume connu.

\section{Préparation de l'échantillon}

On a choisi, pour standardiser au mieux la granulométrie des éléments structuraux et en fonction d'impératifs stricte- ment pratiques (commodités d'humectation et de manipulation à l'état humide), de calibrer entre 1 et $2 \mathrm{~mm}$ les agrégats du matériau étudié. Après séchage à $105^{\circ} \mathrm{C}$, le matériau est broyé et tamisé entre 1 et $2 \mathrm{~mm}$.

Ce choix arbitraire d'une telle classe de diamètre n'affecte pas la généralisation possible des résultats. En effet des travaux antérieurs (BODDAERT, 1976) ont montré que la granulométrie des éléments structuraux n'a pas d'incidence sur l'allure générale du phénomène bien qu'elle détermine en partie la valeur absolue du volume de l'espace poral.

Les échantillons sont humectés par vaporisation jusqu'à obtention du poids d'eau désiré. On laisse ensuite l'humidité s'homogénéiser pendant une semaine, à température constante.

Après une mesure d'humidité de contrôle, on détermine la masse de sol à introduire dans la cellule de compression pour obtenir le $\mathbf{e}_{\mathrm{v}}^{0}$ choisi.

$$
\mathrm{m}=\frac{\rho_{\mathrm{v}} \mathrm{V}_{0}}{\mathrm{e}_{\mathrm{s}}^{0}+\mathrm{e}_{\mathrm{a}}+1}
$$

où $\mathrm{V}_{0}$ est le volume initial de la cellule de compression.

\section{- Choix du matériau:}

Pour que l'espace poral structural soit effectivement la variable traduisant la compacité du matériau, nous avons choisi un matériau tel que le volume de son espace poral textural ne soit pas affecté par l'application de la charge dans la gamme des pressions utilisée (tabl. 1).

Dans le domaine où l'espace poral textural n'est pas saturé, des mesures sur petits agrégats du même matériau avant et après compactage ont montré l'invariance du volume de l'espace poral textural pour des énergies de $590 \mathrm{Kjoules} / \mathrm{m}^{3}$ (BODDAERT, 1976).

Dans le domaine où il est saturé, l'espace poral textural ne varie que sous une pression supérieure à la valeur absolue du potentiel de l'eau qu'il contient. On observerait alors un phénomène de consolidation. Cette considération explique que pour étudier une gamme suffisamment étendue de pression ( 0 à 10 bars) on choisisse un matériau argileux où le potentiel de l'eau reste grand (en valeur absolue) par rapport à la gamme de pression étudiée, même pour des teneurs en eau importante.

\section{RÉSULTATS}

La courbe de compression d'un échantillon saturé, ou courbe de consolidation, traduit les variations de l'indice des vides de cet échantillon liées au départ de l'eau qu'il contient sous l'action de la pression appliquée.

Cette courbe (fig. 1) est généralement assimilée à une droite dans les coordonnées semi-logarithmiques e - Log P (chemin $\mathrm{AE}$ ). Très schématiquement, lorsqu'un échantillon a subi antérieurement une $1^{\text {re }}$ consolidation jusqu'à la

TABLEAU 1

Caractéristiques physiques du matériau utilisé

\begin{tabular}{|c|c|c|c|c|c|c|}
\hline \multicolumn{5}{|c|}{ Granulométrie p. 100} & \multirow{3}{*}{ Densité de solide $\rho_{s}$} & \multirow{3}{*}{ CEC } \\
\hline Argile & \multicolumn{2}{|c|}{ Limons } & \multicolumn{2}{|c|}{ Sables } & & \\
\hline $0-2 \mu$ & $2-20 \mu$ & $20-50 \mu$ & $50-200 \mu$ & $200-2000 \mu$ & & \\
\hline 41,8 & 25,4 & 6,5 & 19,5 & 6,6 & 2,66 & 25 \\
\hline
\end{tabular}
Physical properties of the soil used. 
pression $P_{1}$ (chemin $A B C$ ), on n'observe une reprise de la consolidation que pour une valeur de $\mathrm{P}$ supérieure à $\mathrm{P}_{1}$ (chemin $\mathrm{CDE}$ ). On dit alors que l'échantillon est préconsolidé.

Ce type de comportement attribué classiquement aux échantillons continus se retrouve aussi sur les courbes de compression des massifs d'agrégats exprimées dans les coordonnées $\mathrm{e}_{\mathrm{s}}-\log \mathrm{P}$, quelle que soit leur humidité (fig. 2),

La charge nécessaire à la mise en place de l'échantillon (à l'état initial choisi) dans la cellule de mesure, ou charge de "précompression", est l'homologue de la charge de préconsolidation citée ci-dessus. Elle diminue quand la teneur en eau du massif augmente.

On constate de plus que :

- pour des teneurs en eau inférieure à 10 p. 100 environ, les courbes sont confondues aux erreurs expérimentales près ;

- au-delà, pour un même effort, l'amplitude de la déformation évaluée par une diminution de l'indice des vides augmente avec la teneur en eau.

Les coordonnées $\mathrm{e}_{\mathrm{s}}-\mathrm{W}$ sont plus favorables à la mise en évidence, et à l'analyse des comportements liés à la teneur en eau (fig. 3).

Dans ces coordonnées, à l'équilibre $(t=4 \mathrm{~h})$ et sous charge, les courbes de compression ont l'allure de tridroites définissant 2 seuils hydriques de comportement $\left(W_{1}\right.$ et $\left.W_{2}\right)$ et un point d'arrêt $\left(\mathrm{W}_{3}\right.$ : intersection de la courbe de compression et de l'axe des abscisses).

a) $\mathrm{W} \leqslant \mathrm{W}_{1}$. L'amplitude du tassement est relativement faible. Elle est fonction de l'intensité de la charge appliquée, mais elle est indépendante du temps d'application ainsi que de la teneur en eau du massif. On est, en effet, dans un domaine de teneur en eau où la déformation du massif consécutive à l'enfoncement du piston, d'amplitude d, peut être considérée comme instantanée (fig. 4/a). Le seuil $\mathrm{W}_{1}$ est indépendant de l'intensité de la charge appliquée et correspond à la limite de retrait des agrégats constituant le massif.

b) $\mathrm{W}_{1}<\mathrm{W} \leqslant \mathrm{W}_{2}$. L'amplitude du tassement devient fonction de la teneur en eau mais reste indépendante du temps d'application de la charge (fig. 5). Les pentes des droites dans ce domaine ainsi que la teneur en eau au seuil $\mathrm{W}_{2}$ sont fonction de l'intensité de la charge appliquée. Tout laisse à penser (aux erreurs d'appréciations par lecture graphique près) que $W_{2}$ correspond bien au seuil de

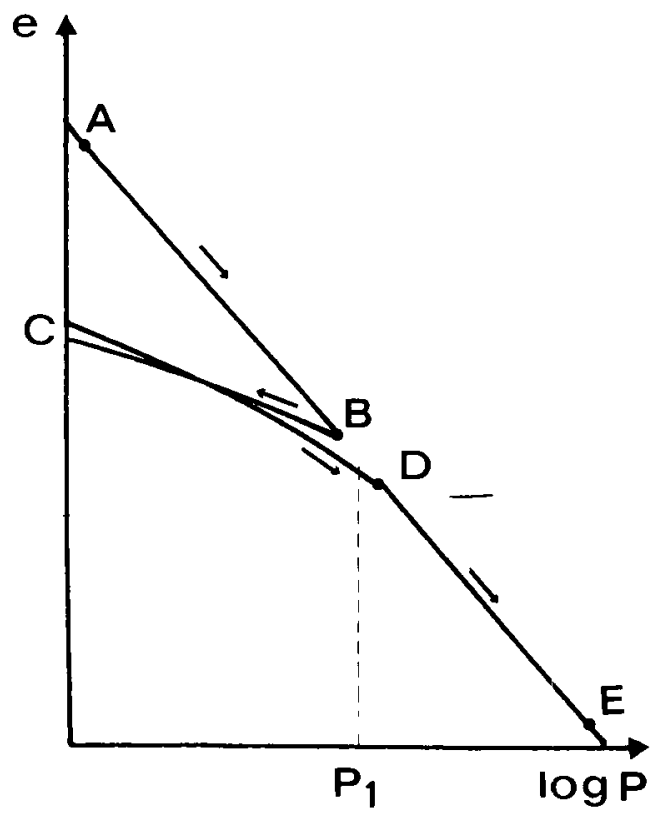

Figure 1

Courbe de compression théorique d'un échantillon quelconque dans des coordonnées semi-logarithmiques.

Idealized compression curve in semi-logarithmic plot.

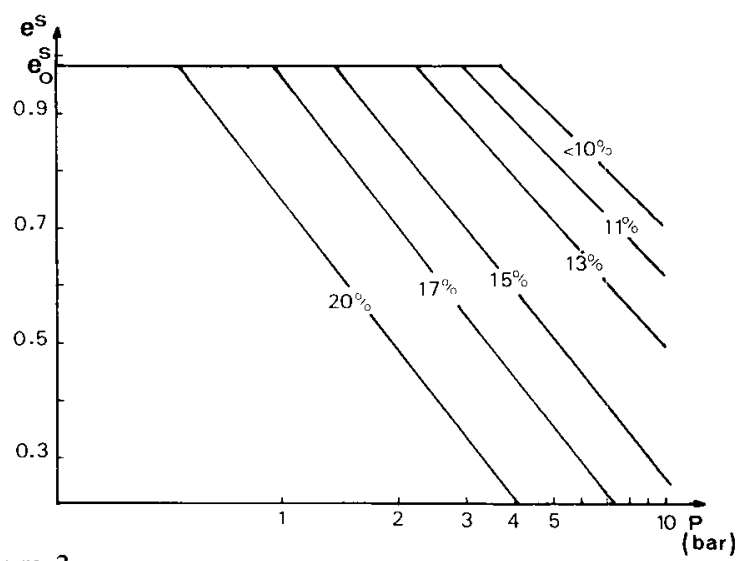

Figure 2

Variations de l'indice des vides structural ( $\left.e^{s}\right)$ en fonction de la pression appliquée $P$ (échelle logarithmique) : Effet de la teneur en eau $(W \%)$.

Relationship between structural void ratio $\left(e^{s}\right)$ and applied pressure $P$ (logarithmic scale): Moisture content effect (W\%).

\section{Figure 3}

Variations de l'indice des vides structural $\left(e^{s}\right)$ en fonction de la teneur en eau (W\%): Effet de l'intensité de la charge $(0,5$ à 10 bars). (Pour la clarté de la figure, les points expérimentaux n'ont été portés que pour les pressions de 1-4-8 bars).

Relationship between structural void ratio $\left(e^{s}\right)$ and moisture content $(W \%)$ : Load intensity effect. (Experimental data were dotted only for 1-4-8 bar pressure, not to overload the figure).

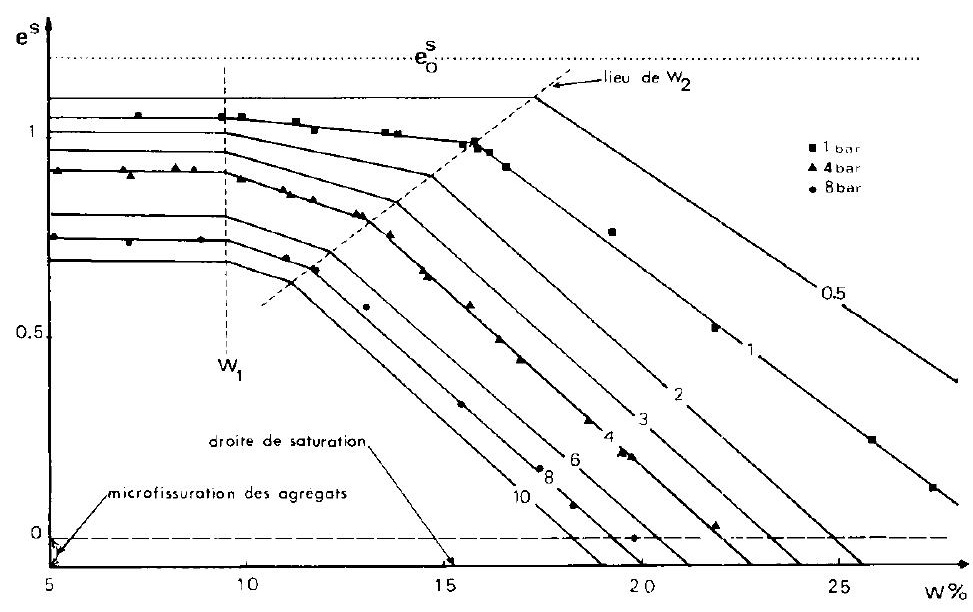


sensibilité au compactage défini par FAURE (1978) sur les courbes obtenues par le test PROCTOR $\left(\rho_{d}=f(W)\right)$ (fig. 5).

c) $\mathrm{W}_{2}<\mathrm{W} \leqslant \mathrm{W}_{3}$. L'amplitude de la déformation dépend du temps d'application (fig. $4 / \mathrm{b}$ et 5) et de l'intensité de la charge.

L'indice des vides structural, évalué à l'équilibre, décroît quand la teneur en eau augmente jusqu'à un point d'arrêt correspondant à la saturation de l'échantillon pour la teneur en eau $W_{3}$. Pour la charge appliquée, le massif est à son maximum de compacité.

Compte tenu de l'allure des courbes, on admettra que ce point d'arrêt peut être obtenu graphiquement en prolongeant la droite de compression jusqu'à son intersection avec la droite de saturation.

Ainsi défini, ce point n'existe que pour des temps d'application de la charge amenant le massif à l'équilibre.

D'autre part, le domaine hydrique dans lequel ces considérations peuvent être admises est borné par la teneur en eau des agrégats amenés à potentiel nul par humectation : au-delà, il n'y a plus identité entre la teneur en eau du massif et la teneur en eau des agrégats.

La définition de ce point d'arrêt, par la saturation de l'éprouvette est la même que celle que donne FAURE (1978) du maximum de compacité de la courbe PROCTOR (fig. 5). La détermination graphique de $\mathrm{W}_{3}$ dépend évidemment du choix des coordonnées dans lesquelles est représentée la courbe de compression.

\section{DISCUSSION ET CONCLUSION}

Pour interpréter ces résultats, acquis sur des matériaux discontinus, il faudrait pouvoir faire appel à des théories de la mécanique des milieux granulaires. Etant donné la complexité des phénomènes, les recherches actuelles en ce domaine portent essentiellement sur des milieux dont les grains sont solides et non déformables.

Dans ce travail, par contre, les grains ou agrégats composant les massifs étudiés sont fragiles et déformables. Pour analyser les déformations dans de tels échantillons, il faut donc faire intervenir :

- Les propriétés mécaniques du massif résultant des contacts entre agrégats,

- Les propriétés mécaniques des agrégats eux-mêmes.

C'est ainsi que FAURE (1978) est amené à interpréter les deux premières parties de la courbe PROCTOR:

- avant le seuil de sensibilité au compactage $\mathrm{W}_{\mathrm{s}}$, il y a concassage et réarrangement des agrégats,

- après le seuil $\mathrm{W}_{\mathrm{s}}$, les agrégats deviennent déformables avec l'entrée en plasticité de la phase argileuse.

De même, BraunaCK \& DEXTER (1978) utilisant, entre autres, les travaux de ROGOWSKI et al. (1968) font intervenir dans leur modèle la cohésion propre aux agrégats dans la compressibilité de l'ensemble du massif.

On pourra distinguer 2 niveaux d'analyse qui seront, si le comportement du matériau satisfait au critère de Coulomb :

- Les caractéristiques mécaniques résultantes de l'assemblage des éléments constitutifs du matériau : cohésion des agrégats, $C_{a}$; angle de frottement interne intra agrégats, $\varphi_{\mathrm{a}}$.

- Les caractéristiques mécaniques résultantes de l'assemblage des éléments structuraux : cohésion du massif d'agrégats, $\mathrm{C}_{\mathrm{s}}$; angle de frottement interne inter-agrégats, $\varphi$ s.

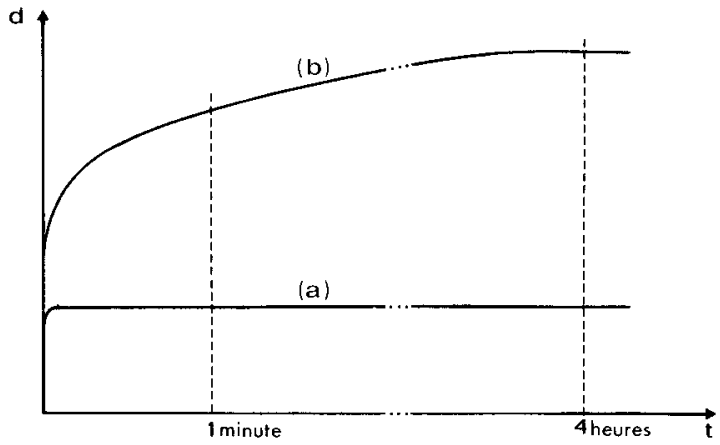

Figure 4

Influence du temps d'application de la charge sur l'amplitude de la déformation (d).

a) déformation instantanée : $W<W_{2}$

b) l'amplitude de la déformation dépend du temps d'application de la charge: $\boldsymbol{W}>W_{2}$

Time-deformation diagram :

a) instantaneous deformation $W<W_{2}$

b) time-dependent deformation $W>W_{2}$

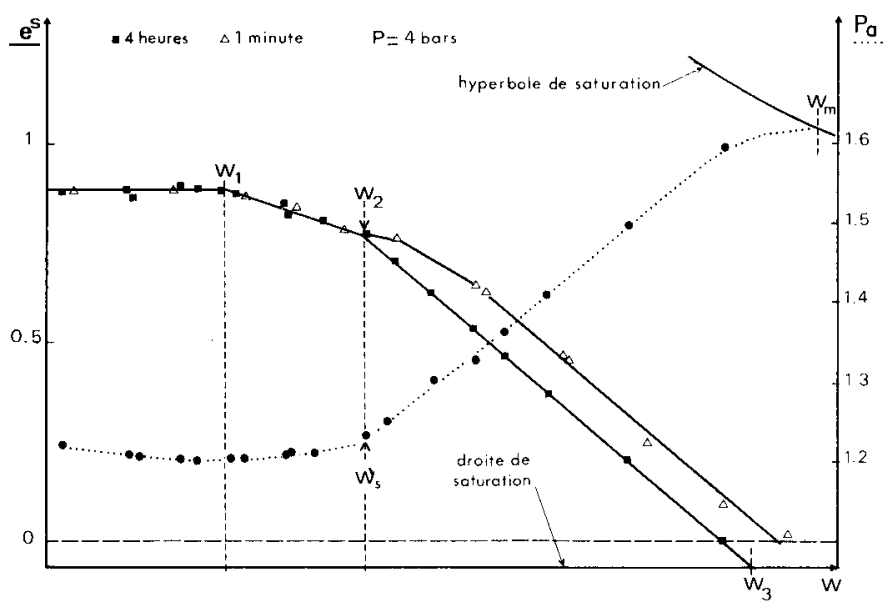

Figure 5

Comparaison des courbes de compactage exprimées soit en terme de variations de l'indice des vides structural $\left(e^{s}\right)$, soit de variations de la densité $\rho_{d}$. Effet du temps d'application de la charge.

Comparison between compaction curves expressed either as variations of structural void ratio or as variations of bulk density. Effect of loading time.

Ainsi Collis-GeORge \& Lloyd (1978), cherchant à estimer les contraintes de cisaillement d'un lit de semences, préfèrent évaluer les frottements entre agrégats que les contraintes de cisaillement propres aux agrégats. C'est ainsi qu'ils observent in situ des angles de frottement interne de l'ordre de $65^{\circ}$. De telles valeurs, nettement supérieures à celles obtenues classiquement sur un sable sec $\left(\varphi=42^{\circ}-45^{\circ}\right)$ sont aussi mentionnées par K. HARTGE (1974) dans son travail sur les cisaillements de massifs d'agrégats. Ce rôle déterminant attribué à $\varphi_{s}$ est très dépendant de $W$.

Nous avons pu constater, pour notre part, en cisaillant à la boîte de Casagrande des massifs d'agrégats de kaolinite ( $\varnothing=2 \mathrm{~mm}$ (fig. 6), le comportement de pseudo-sable de ces agrégats pour les faibles teneurs en eau :

- cohésion élevée des agrégats mais cohésion nulle du massif,

- angle de frottement faible à nul dans le matériau continu mais angle de frottement interne élevé entre agrégats. 


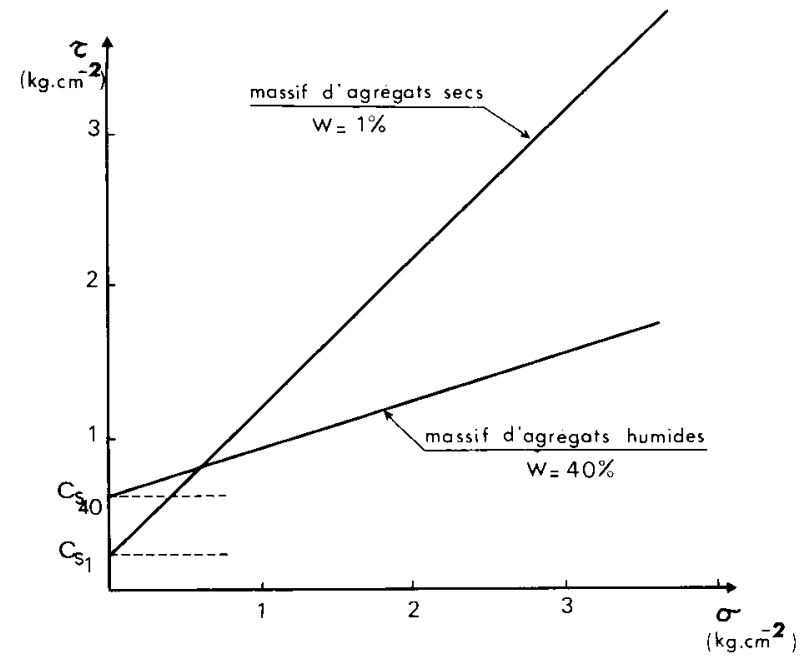

Figure 6

Courbes de cisaillement (droites de Coulomb) de massifs d'agrégats de kaolinite: Effet de la teneur en eau.

Shearing curves (Coulomb's criterion) of aggregate beds (kaolinite) : Moisture content effect.

Mais quand la teneur en eau augmente, la cohésion et l'angle de frottement interne de l'ensemble du massif tendent respectivement vers la cohésion et l'angle de frottement interne propres aux agrégats.

A partir de ces éléments, certes fragmentaires, on peut tenter d'interpréter comme suit le mécanisme de tassement d'un massif d'agrégats.

a) $\mathrm{W}<\mathrm{W}_{1}$. Le comportement des agrégats est celui de pseudo-sables. Leur réarrangement s'effectue, sans qu'ils soient eux-mêmes affectés par la charge qu'ils subissent : leur cohésion prore $\mathrm{C}_{\mathrm{a}}$ serait alors supérieure aux contraintes auxquelles ils sont soumis.

On a constaté, en effet, que des compressions simples sur des échantillons remaniés, de texture voisine de celle de notre matériau, pour une structure continue et dans cette même gamme de teneur en eau donne des résistances à la compression simple de l'ordre de 100 à $150 \mathrm{~kg} / \mathrm{cm}^{2}$ (FIES, comm. pers.). L'angle de frottement inter-agrégats, $\varphi_{s}$, déterminerait donc en grande partie ces mécanismes de tassement dans ce domaine de teneur en eau.

b) $\mathrm{W}_{1}<\mathrm{W}<\mathrm{W}_{2}$. L'influence de la teneur en eau sur l'amplitude du tassement pourrait s'expliquer par une diminution importante de la cohésion $\mathrm{C}_{\mathrm{a}}$ et/ou une variation de l'angle de frottement interne inter-agrégats, $\varphi_{s}$, pour un accroissement de la teneur en eau.

- La cohésion $\mathrm{C}_{\mathrm{a}}$ serait alors de l'ordre de grandeur des contraintes se développant dans le massif. La fragmentation des agrégats (rupture dite fragile) sans remaniement de l'assemblage des constituants, suivie de leur réarrangement pourrait expliquer une augmentation de compacité.

- Ce réarrangement des agrégats pourrait par ailleurs être favorisé par une diminution de $\varphi_{\mathrm{s}}$.

c) $\mathrm{W}_{2}<\mathrm{W}<\mathrm{W}_{3}$. Lors de la compression, l'angle de frottement interne du massif d'agrégats $\varphi$ serait alors une combinaison entre les angles de frottement interne inter et intra-agrégats : $\varphi=\mathrm{f}\left(\varphi_{\mathrm{a}}, \varphi_{\mathrm{s}}\right)$, la cohésion $\mathrm{C}_{\mathrm{a}}$ déterminant en partie le niveau de la déformation enregistrée. FAURE (1978) a montré en effet la décroissance brutale de l'angle de frottement interne $\varphi$ d'une éprouvette obtenue par compactage PROCTOR à partir du seuil $\mathrm{W}_{2}$.

Mais en plus, dans cette gamme de teneur en eau, on ne peut plus considérer la déformation comme iristantanée : le comportement des agrégats sous charge est alors viscoélastique. L'amplitude de la déformation est en effet fonction du temps d'application de la charge.

Ce type d'interprétation du comportement au compactage des milieux discontinus devrait pouvoir être généralisé à différents types de matériaux.

- d'une part, en développant la notion de caractéristiques mécaniques intrinsèques en reliant la cohésion et l'angle de frottement interne au niveau textural à des variables de constitution.

- d'autre part, en reliant les comportements mécaniques et les variables qui les décrivent au potentiel de l'eau dans le matériau en cours de compression.

L'expression des teneurs en eau du matériau en potentiel permettra de prendre en compte les interactions entre " contrainte hydrique " et "contrainte mécanique " dans le comportement au compactage et de rendre comparables les seuils hydriques de changement de comportement de matériaux de texture variées.

L'utilisation de test de compactage de laboratoire ne donnera en aucun cas les moyens de prévoir directement le comportement au compactage des couches de sols en place. Mais elle est un préalable nécessaire à la généralisation d'études de terrain visant à prendre en compte, dans des situations bien typées, les anisotropies de constitution (matières organiques), d'organisation et d'hydratation des couches de surface dans les phénomènes de tassement.

La confrontation des caractéristiques mécaniques intrinsèques des matériaux avec des observations plus globales de comportement in situ devrait permettre de dégager des règles, à la fois analytiques et empiriques, de prédiction du comportement d'une couche de sol de surface et donc:

- de choisir des conditions d'états physiques favorables aux opérations culturales visées,

- d'envisager des solutions techniques au niveau des choix, voire des conceptions des matériels agricoles.

Reçu le 22 avril 1981. Accepté le 18 novembre 1981.

\section{RÉFÉRENCES BIBLIOGRAPHIQUES}

Boddaert D., 1976. Etude du comportement mécanique d'un sol travaillé soumis au roulage des engins agricoles. Mémoire de fin d'études. E.N.I.T.A. Dijon-Quetigny.

Braunack M. V., Dexter A. R., 1978. Compaction aggregate beds, p. 119-126, in Emerson Bond. Dexter (Editors) Modification of soil structure, J. Wiley, Chichester, $438 \mathrm{p}$.

Chancellor W. J., 1977. Compaction of soil by agricultural equipment. Bulletin 1881, Div. of Agricultural Sciences, Univ. of California.
Collis-George N., Lloyd J. E., 1978. Description of seed beds in terms of shear strength, p. 111-117, in Emerson Bond. Dexter (Editors) Modification of soil structure, J. Wiley, Chichester, $438 \mathrm{p}$. Devaud D., 1977. Contrôle, réalisation et traitement automatiques d'essais de laboratoire en Mécanique des sols: Essais triaxiaux et aedométriques, Thèse, doct.-ing. E.N.S.A. de Rennes, 160 p.

Eriksson J., Håkansson I., Danfors B., 1974. The effect of soil compaction on soil structure and crop yields. Bull. 354. Swed. Inst. of agric. Engineer., 101 p. 
Faure A., 1978. Comportement des sols au compactage : Rôle de l'argile et conséquences sur l'arrangement des grains. Thèse Doct. ès Sci. Grenoble, $179 \mathrm{p}$.

Fies J. C., 1978. Porosité du sol, étude de son origine texturale. Thèse, Doct. ès Sci. U.L.P., Strasbourg, 145 p.

Fies J. C., Stengel P., 1981. Densité texturale de sols naturels. Agronomie, 1 (8), 651-666.

Guerif J., Faure A., 1979. Rôle de la matière organique sur le comportement des sols au compactage : I. Etude statistique. Ann. agron., 30 (5), 387-399.

Guerif J., 1979. Rôle de la matière organique sur le comportement des sols au compactage : II. Matières organiques libres et liées. Ann. agron., 30 (6), 469-480.

Hartge K. H., 1974. Trans of tenth. Intern. Congr. Soil. Sci. 1, 194201.
Kuipers H., 1959. Meded. Landbouwhogesch. Opzockingsstn staat. Gent. 24, 349-355.

Rogowski A. S., 1964. Strength of soil aggregates. Ph. D. Thesis lowa State University.

Rogowski A. S., Moldenhauer W. C., Don. Kirkham, 1968. Rupture parameters of soil aggregates. Soil Sci. Soc. Am. Proc. 32, 720724.

Scott Blair G. W., 1937. Compressibility curves as a quantitative measure of soil tilth. 1. J. agric. Sci., 27, 541-556.

Scott Blair G. W., Cashen G. H., 1938. Compressibility curves as a quantitative measure of soil tilth. 2. J. agric. Sci., 28, 367-378.

Stengel P., 1979. Utilisation de l'analyse des systèmes de porosité pour la caractérisation de l'état physique du sol in situ. Ann. agron., 30 (1), $27-51$. 